\title{
Reforming Healthcare for Former Prisoners
}

\author{
Elizabeth S. Barnert, MD, MPH, MS $S^{1,2,3}$, Raymond Perry, MD, MSHS , \\ and Kenneth B. Wells, MD, MPH $H^{1,3,5,6,7}$
}

${ }^{1}$ Robert Wood Johnson Foundation Clinical Scholars Program, UCLA, Los Angeles, CA, USA; ${ }^{2}$ Departments of Pediatrics and Medicine, David Geffen School of Medicine, UCLA, Los Angeles, CA, USA; ${ }^{3}$ Department of Health Policy and Management, UCLA Jonathan and Karin Fielding School of Public Health, Los Angeles, CA, USA; ${ }^{4}$ County of Los Angeles Department of Health Services, Juvenile Court Health Services, Los Angeles, CA, USA; ${ }^{5}$ RAND Corporation, Santa Monica, CA, USA; ${ }^{6}$ Departments of Psychiatry and Biobehavioral Sciences, David Geffen School of Medicine UCLA, Los Angeles, CA, USA; ${ }^{7}$ Center for Health Services and Society, UCLA Jane and Terry Semel Institute for Neuroscience and Human Behavior, Los Angeles, CA, USA.

KEY WORDS: former prisoners; former inmates; health care reform; Affordable Care Act.

J Gen Intern Med 29(8):1093-5

DOI: $10.1007 / \mathrm{s} 11606-014-2816-y$

(c) Society of General Internal Medicine 2014

\section{THE AFFORDABLE CARE ACT \& FORMER PRISONERS}

The Affordable Care Act (ACA), if successfully implemented, offers new opportunities to provide appropriate care to a vulnerable and marginalized community-former prisoners. Currently, 2.3 million American adults are incarcerated, representing approximately one in 100 adults. ${ }^{1}$ Most will be released. While incarceration rates are rising in some states and leveling off in others, release rates are generally increasing, largely due to policy changes dictating management of less serious offenders. ${ }^{2}$ How will the ACA and other federal policies such as the Mental Health Equity and Addiction Parity Act (MHEAPA) impact this large, medically and socially vulnerable population?

Before 2014, most former prisoners lacked health insurance. ${ }^{3}$ This can be expected to change with healthcare reform. Approximately 730,000 prisoners are released from federal prisons each year. Under the ACA, $23.5 \%$ more than 172,000 former prisoners annually - would be eligible for tax credits to assist with purchasing insurance. Additionally, if all states accept the ACA's Medicaid expansion, an estimated 245,000 newly released prisoners would be eligible to enroll in Medicaid each year. ${ }^{2}$ Even if Medicaid expansion is limited only to the states that currently plan to expand, roughly 100,000 newly released prisoners are expected to enroll in Medicaid each year (estimated by the authors from the Kaiser Family Foundation's state-by-state Medicaid enrollment projections). When one considers that over 9 million inmates are released annually from jails and prisons, the number eligible for coverage on release each year may be in the millions. How will healthcare systems and providers prepare for this

Published online March 8, 2014 population of former inmates? What are their unique needs? What are clinician tasks and responsibilities?

Incarceration disproportionately affects young, poor men and racial and ethnic minorities. One in 15 black men are incarcerated at any given time, compared to one in 36 Hispanic men, and one in 106 white men. While men are about ten times as likely to be in prison as women, incarceration rates among women are rising and demonstrate similar racial disparities. ${ }^{1}$

Former prisoners are more likely than others to be sick. An estimated $80 \%$ of recently released males and $90 \%$ of females have chronic medical, mental health, or substance use problems. ${ }^{3}$ In contrast to the general population prevalence of hepatitis B, C, and HIV of approximately $1 \%$ each, in one sample of returning prisoners, $11 \% \mathrm{had}$ hepatitis B or C, $5 \%$ had tuberculosis, and $2 \%$ had HIV/ AIDS. $^{3}$

Given the substantial morbidities in this population, former prisoners could derive significant benefit from access to affordable healthcare. Despite high rates of uninsurance, one study of former prisoners demonstrated that over $70 \%$ utilized healthcare services within 10 months following release. Treatment rates, however, declined as most were using acute, episodic care. ${ }^{3}$ The expansion of Medicaid in some states will be important, but ensuring that healthcare reform reaches and improves care for former prisoners will require additional steps.

\section{POLICY RECOMMENDATIONS}

Achieving continuity of care for former prisoners would require several policy changes:

- First, to prevent delays in care related to insurance status after prison release, states could suspend rather than terminate Medicaid benefits during incarceration. ${ }^{2}$

- Second, to expedite enrollment, Medicaid eligibility determinations, which require data on vital statistics, employment status, and income, could, with adequate privacy protections, incorporate such data from corrections systems. ${ }^{2}$ 
- Third, policies that facilitate enrollment in Medicaid or exchanges prior to or at release would likely be beneficial. ${ }^{4}$ For example, Massachusetts increased insurance uptake among newly released prisoners from $40 \%$ to $90 \%$ by implementing a "virtual gateway" Medicaid application that allowed prisoners to leave with coverage. ${ }^{4}$

- Fourth, policies supporting targeted outreach to individuals with chronic medical and behavioral health problems may help improve health outcomes. Treating behavioral health problems will be more feasible as the ACA and the MHEAPA assure coverage at parity for behavioral health conditions in expanded Medicaid and the exchanges.

- Fifth, policies that eliminate barriers for former prisoners to gain employment or to live with families in supported housing may decrease exposure to poverty and homelessness.

Physicians can learn how their states are addressing these issues and can advocate on their patients' behalf.

\section{PHYSICIANS PRACTICING IN ACCOUNTABLE CARE ORGANIZATIONS (ACOS) \& MEDICAL HOMES}

The ACA promotes models of coordinated care. One of the challenges that physicians practicing in ACOs and medical homes will face is that many former prisoners may become repeatedly incarcerated and released, threatening continuity of care. During an individual's reentry into the community, a physician practicing in a medical home is in an excellent position to coordinate care and address health risks encountered prior to, during, and after their patient's release. For patients with severe mental illness or addictive disorders, physicians may find health homes, which integrate primary care into traditionally behavioral health settings, a promising alternative to the medical home model. Under either model, the primary care physicians' coordination of medical care with behavioral health providers and social workers to address factors such as unemployment and social isolation will likely prove essential. In this way, physicians can take the lead in promoting effective treatment and potentially even help to prevent recidivism.

Additionally, collaboration with trusted communitybased agencies, such as faith-based programs and community centers, to jointly address healthcare and social reintegration goals, including housing, employment, and family reunification, may be especially beneficial for former prisoners. A recent randomized trial demonstrated that such an integrated approach improved health and social outcomes for underserved depressed clients, including former prisoners. ${ }^{5}$ For medical home patients who become incarcerated, primary care providers can facilitate exchange of diagnostic or treatment information with the correctional system, which may extend benefits of the medical/health home model to the correctional setting.

\section{PROVIDER RESPONSIBILITIES}

Healthcare providers who practice in communities with higher rates of incarceration might better serve their patient population by becoming more knowledgeable about the prevalence and health impacts of incarceration, as well as the social contexts of former prisoners' lives on release. Initiating appropriate care may require screening for prison exposure and resulting health risks. Such a history need not ask about specific charges or presumed innocence or guilt. Similarly, a history of incarceration in family members is relevant social history. Although some might consider such screening as stigmatizing, incarceration is as clinically relevant as other sensitive but important health risk factors, such as gun ownership, sexual orientation, or drug use.

Healthcare providers may want to become more knowledgeable about former prisoners' communicable disease risks, mental health needs, and substance use issues, as well as the health-related social consequences of incarceration. A discussion about post-release employment, housing security, and social support is paramount to addressing patients' overall health and stability. Doctors can learn about the expanded benefits offered under the ACA and MHEAPA, such as parity of mental health and addiction coverage as essential benefits in exchanges and expanded Medicaid. Perhaps many physicians feel that treating former prisoners is not within their scope of practice. With many former prisoners gaining coverage, it will become important for doctors generally to expect to treat and, as needed, advocate for these patients as an especially vulnerable community. Recently released prisoners, the majority of whom currently lack basic insurance coverage, tend to be heavy users of higher-cost acute care services such as hospital admissions and emergency room visits. ${ }^{4}$

\section{CONCLUSION}

The ACA coincides with efforts to release less serious offenders, increasing the number of former inmates requiring services. Medicine will need to establish an approach to caring for former prisoners that acknowledges their unique clinical and social history, while accepting and normalizing the provision of high quality, comprehensive care. While the ACA helps assure basic insurance coverage, achieving appropriate access requires that healthcare systems are organized and providers are trained to meet the needs of this vulnerable population. 
Acknowledgements: Contributors: We thank Loretta Jones and Drs. Robert Brook and Paul Chung.

Funders: RWJF funded Dr. Barnert. NIMH and PCORI supported Dr. Wells. The opinions expressed are those of the authors.

Prior Presentations: This article has not been presented at any conferences.

Conflict of Interest: The authors declare that they do not have a conflict of interest.

Corresponding Author: Elizabeth S. Barnert, MD, MPH, MS; Robert Wood Johnson Foundation Clinical Scholars Program, UCLA, Los Angeles, CA, USA (e-mail: ebarnert@mednet.ucla.edu).

\section{REFERENCES}

1. Warren J, Gelb A, Horowitz J, Riordan J. One in 100: Behind Bars in America 2008. Washington DC: The Pew Center on the States; 2008.

2. Cuellar AE, Cheema J. As roughly 700,000 prisoners are released annually, about half will gain health coverage and care under federal laws. Health Aff (Millwood). 2012;31(5):931-938.

3. Mallik-Kane K, Visher C. Health and Prisoner Reentry: How Physical, Mental, and Substance Abuse Conditions Shape the Process of Reintegration. Washington DC: The Urban Institute Justice Policy Center; 2005.

4. Bainbridge A. The Affordable Care Act and Criminal Justice: Intersections and Implications. Washington DC: Bureau of Justice Assistance U.S. Department of Justice; 2012.

5. Wells KB, Jones L, Chung B, et al. Community-Partnered ClusterRandomized Comparative Effectiveness Trial of Community Engagement and Planning or Resources for Services to Address Depression Disparities. J Gen Intern Med. 2013;(10):1268-1278. 